\title{
A commentary on Micah Bregman et al.: A method for testing synchronization to a musical beat in domestic horses (Equus ferus caballus)
}

\author{
SANDY S. VENNEMAN \\ University of Houston-Victoria
}

\begin{abstract}
This commentary provides additional information related to equines and suggestions for strengthening the proposed protocol for testing synchronization to a musical beat in this species.
\end{abstract}

Submitted 2012 November 8; accepted 2012 November 8.

KEYWORDS: equine perception, synchronization, nuisance variables

\section{INTRODUCTION}

THE authors propose a methodology to explore the vocal learning and rhythmic synchronization hypothesis put forward by Patel (2006) in domestic horses that predicts that horses are incapable of rhythmic synchronization because they are vocal non-learners. However, anecdotal accounts of horses spontaneously entraining to musical beats abound in equestrian circles. Since there is no scientific study of rhythmic synchronization, the authors propose a new experimental method which they term "circular trotting to music", or CTM, to allow for such scientific investigation. The authors explain that if horses are capable of musical beat perception and synchronization (BPS) the vocal learning and rhythmic synchronization hypothesis is not supported. Should such experimental data in horses be produced, it would open a dialog regarding what substrates are central to this ability.

Their paper describes an experimental procedure to test if horses are capable of BPS. My commentary will address this described protocol in an effort to minimize nuisance variables that may impact the ability of researchers to find BPS if it occurs and provide equine terminology that is used internationally.

\section{PRIOR RESEARCH ON HORSES AND MUSIC AND PROPOSED RESEARCH PROTOCOL}

\begin{abstract}
Audition
Perceptual abilities of the test animal, in this case the horse, must be addressed. If the stimulus falls outside the perceptual abilities the experiment is invalid. The authors describe the auditory capabilities of horses under the heading "prior research on horses and music." Heffner (1992) is cited and an audiogram is displayed that compares human and horse hearing thresholds. In a more recent paper, Heffner (1998) notes that horses have an auditory range from 55 to $33,500 \mathrm{Hertz}(\mathrm{Hz})$ and their best sensitivity is at a frequency of $2,000 \mathrm{~Hz}$ and 7 decibels $(\mathrm{dB})$. Given this information, the proposed protocol could be strengthened if both variables of frequency and intensity are experimentally controlled. Currently the protocol calls for the music to be played over the speakers in an indoor arena, using speakers with a range of $58 \mathrm{~Hz}$ to $18,500 \mathrm{~Hz}$ but makes no mention of measurement of the intensity $(\mathrm{dB})$ of music. Measuring and adjusting the decibels of trial music at the level of the test horse's ears would allow for consistency across multiple trials with the same animal, or across animals in different environments.

The proposed protocol calls for the use of an indoor arena to decrease external stimuli and thereby help control for the possibility of the horse attending to sounds other than the independent variable of music. The indoor arena could also minimize visual differences in the experimental environment that may confound results (Heffner, 1998).
\end{abstract}


The authors note that the case study uses a gelding. This would minimize the impact of sex hormones in this one experiment, but future researchers may want to include sex as a variable since hormones have been shown to impact audition in multiple species (Al-Mana et al., 2008).

\section{Vision}

Visual stimuli can also impact the behavior of horses. One of the earliest psychological experiments involved a horse named Clever Hans, who responded to very subtle cues from his handler by pawing the answer to math questions. When the person asking the math question did not know the answer, or was hidden from Clever Hans' view, he could no longer answer the question correctly. Thus the impact of almost imperceptible signals was documented and has been since called the "Clever Hans Effect." Hanggi and Ingersoll (2012) demonstrated that horses have visual abilities of nearly 360 degrees, so the proposition that the controller was not in the view of the horse for one third of the circle in the case study is likely to be inaccurate. However, the researchers have controlled for this confound in their current proposed protocol by hiding equipment controllers from the horse. Perhaps, more importantly, the authors eliminate inadvertent rhythmic cues from the horse handler by proposing the use of headsets supplying sounds that have no beat.

\section{The circular trotting to music (CTM) method}

The authors of this research need a procedure that would eliminate as much human-horse communication as possible. With a mounted rider the horse can respond to multiple cues in addition to the auditory one supplied by the experimenter. Educated equestrians use all four aids to communicate with their mounts: hands, legs, seat, and voice. The seat (weight distribution and movement) is particularly influential as a horse will follow the rhythm of a rider if that rhythm is not synchronized with their own. The size of the horse's stride is also influenced by a rider with an educated seat. This influence allows the rider to influence the rhythm of the horse's footfalls. The difficulty of using a mounted rider arises when the rider unknowingly influences the movement of their mount with the aids. To eliminate seat and leg aids, the authors employ an un-mounted technique termed lunging. They minimize the impact of the hands by calling for a loose contact with the horse, and eliminate the voice by having the handler remain silent during data collection.

The training of horses has been described in text since ancient times starting with Xenophon 430-354 BC (Xenophon, 1925). The current study protocol would be strengthened if it used classical methods of training and terminology that are understood by equestrians internationally. In the proposed protocol, the authors describe a procedure that is classically termed lunging, in which the horse moves around the handler on a rope. This rope is generally termed a lunge line, rather than the stated "lead line." A lead is shorter and is used to lead a horse, not for groundwork. In the same paragraph they describe the lunge line placing the horse ten feet from the handler. This distance is unsafe, as a horse may show exuberance on the lunge and kick out, potentially injuring the handler. In addition, a circle of such small radius will inhibit the movement of most horses, except for very small ones, or ones with minimal stride (length of steps). Common practice in lunging is to spend the majority of the session on a 20 meter circle with the horse being ten meters from handler instead of ten feet. Likewise using traditional equipment and terminology would increase confidence in the procedure. A lunge whip would be used in place of the terminology in the protocol, "carrot stick and string" (Bryant, 2006).

Potential researchers could investigate the impact of the lunging technique by replicating with circles of varying sizes and assessing their influence on the ability of the horse to use its' body and entrain to the musical rhythm. The authors also note they conducted some trials off the lunge line. This variation would help eliminate any unintentional communication from the handler through the lunge line. This modification could call for the use a lunging pen, also called a round pen, if it were of ample size to avoid restricting movement of the horse. The horse in the case study was a Morgan and appears to be fairly typical of the breed, being small and compact. Even with his small size, he is leaning toward the center of the circle, and falling on the forehand. Both indicate a lack of balance that may be decreased if the procedure employed a larger circle.

A likely subject pool for further study would come from the discipline of horsemanship termed dressage, which is the French word for training. Any horse can be improved through dressage training, but some breeds have been selectively bred with qualities to fit the demands. These include warmbloods that are typically larger than the case study Morgan. Increasing the size of the circle will enable horses with a larger stride to remain in balance and move naturally, as well as small ones. Additionally, on a less 
restrictive circle, the time the horse remains in motion may increase, allowing for more trials to have sufficient data for coding.

Dressage riding has a long history of using music in training, exhibition, and competition. Musical rides are part of dressage competitions nationally, internationally, and are showcased at the Olympic Games. The US National level governing body for dressage, The United States Dressage Federation, sets rules for scoring rides to music, termed musical freestyles. The current rulebook instructs the judge to reward how well the music expresses the gaits of the horse and the appropriateness of the rhythm and tempo of the music to the horse (USDF Member Guide, 2011). The owner of the horse picked the music in the case study presented by the authors. Experimental validity would suffer if each horse were tested to different music in the experimental protocol. However, a strength of the proposed musical protocol is insuring that the test music chosen has a strong beat and that each horse has the physical ability to trot to the tempo chosen. The authors propose to use the same music and mechanically alter the tempo for different trials also strengthing the protocol.

The protocol also states that the handler should "maintain eye contact" with the horse. Horses respond to non-verbal cues, such as gaze, from other horses as well as handlers. Facing the horse obliquely toward the rear, with the line of sight on the hindquarters encourages forward movement in a horse, while obliquely facing toward the head, with the line of sight to the horses eye, is a signal to stop or slow. Therefore, unspecified gaze could add unwanted variation to trials. To reduce this variance, a simple change to the protocol could create stability over trials and across participants. Placing an adhesive target on the horse's hip and instructing the handler to look at the "target" could minimize variation due to this non-verbal cue, as well as promote longer sets of trotting for sampling.

\section{Data analysis method}

The protocol description of recording the horse's footfalls includes video, as in the case study, and a proposal to alternatively use an accelerometer. The procedure calls for multiple researchers to code footfalls frame by frame with the sound turned off. This eliminates the potential threat to validity from experimenter expectancies that would manifest as coders unconsciously finding synchronization when there is none (Cook \& Campbell, 1979).

Humans show a strong tendency to favor the use of one hand over the other "handedness" and also exhibit "footedness" from a young age (Berger et al., 2011). Just like humans, horses exhibit a preferred or dominant foot (Lucidi et al., 2013). Most horses will use their dominant foot to start an upward transition (changing gait) if not cued by a rider. For this reason it might be beneficial to look at the beat on the dominant forelimb in addition to the current protocol of counting the footfalls of both forelimbs.

\section{DISCUSSION}

The authors note that there are many breeds of horses and this may impact results. Horses of different breeds generally have different temperaments and energy levels that could influence trials if not considered and controlled through experimental procedures. For example, will the trials be run before the horse is exercised for the day or after? Often horses "blow off steam" at the start of a training session and pay less attention during this period than after they have settled down. It might be advisable to allow the horse a specified warm-up period before collecting data or operationalize warmed-up as the point that the horse voluntarily stops voluntary movement. In addition to variation between breeds of horse, there is considerable variation within breeds. We might logically extrapolate from humans that some horses may have "musical inclinations" while others do not. Therefore a fairly large sample size would be necessary to adequately investigate this issue insuring the sample is not composed of only horses "with two left feet."

In closing, the authors have suggested an interesting experimental design, CTM, to test if anecdotal accounts of horses exhibiting BPS can be supported. If BPS is experimentally supported in horses the vocal learning and rhythmic synchronization hypothesis (Patel, 2006) will need revision.

\section{NOTES}

[1] Corresponding author. Departments of Psychology \& Biology, University of Houston-Victoria, 3007 North ben Wilson, Victoria, TX 77995. Email: vennemans@uhv.edu 


\section{REFERENCES}

Al-Mana, D., Ceranic, B., Djahanbakhch, O., \& Luxon, L.M. (2008). Hormones and the auditory system: A review of physiology and pathophysiology. Neuroscience, Vol. 153, No. 4, pp. 881-900.

Heffner, H.E. (1998). Auditory awareness. Applied Animal Behaviour Science, Vol. 57, pp. $259-268$.

Berger, S.E., Friedman, R., \& Polis, M.C. (2011). The role of locomotor posture and experience on handedness and footedness in infancy. Infant Behavior and Development, Vol. 34, No. 3, pp. 472-480.

Bryant, J.O. (2006). The USDF Guide to Dressage. North Adams, MA.: Storey Publishing.

Cook, T.D., \& Campbell, D. T. (1979). Quazi-experimentation: Design \& Analysis Issues for Field Settings. Boston: Houghton Mifflin Company.

Hanggi, E.B., \& Ingersoll, J.F. (2012). Lateral vision in horses: A behavioral investigation, Behavioural Processes. Vol. 91, No. 1, pp. 70-76.

Lucidi, P., Bacco, G., Sticco, M., Mazzoleni, G., Benvenuti, M., Bernabo, N., \& Trentini, R. (2013). Assessment of motor laterality in foals and young horses (Equus caballus) through the analysis of derailment at trot. Physiology \& Behavior, Vol. 109, pp. 8-13.

Patel, A.D. (2006). Musical rhythm, linguistic rhythm, and human evolution. Music Perception, Vol. 24, pp. 99-104.

Pfungst, O. (1911). Clever Hans: A Contribution to Experimental Animal and Human Psychology (Translated by Rahn, C.L.). New York: Henry Holt and Company.

USDF Member Guide (2011) www.usdf.org

Xenophon (1925). Scripta Minora and Constitution of the Athenians. (E. C. Marchant \& G.W. Bowersock, Trans.) Cambridge, MA: Harvard University Press. 